\title{
Safety and Efficacy of an Outpatient Intrathecal Catheter Trialing Method for Targeted Drug Delivery
}

\author{
Vipul Mangal MD, Maged Hamza MD, and Michael Harned MD
}

Background: Chronic pain is a significant health challenge, and targeted drug delivery (TDD) is increasingly utilized to manage patients who have failed several treatment modalities. An intrathecal trial is mandatory prior to permanent implantation, with current guidelines recommending inpatient observation of 23 hours or inpatient admission for a continuous intrathecal infusion opioid trial.

Objectives: TDD is an established method of the delivery of opioids, and in this study, we aim to provide a safe methodology using an outpatient protocol.

Study Design: The study is designed as a retrospective analysis on all non-malignant chronic pain patients who were candidates for TDD, who underwent an outpatient catheter trial from January 1, 2015 to December 31, 2016.

Setting: The study took place in an outpatientbased community practice.

Methods: A retrospective chart review and patient experience questionnaire were utilized to assess the safety and efficacy of 98 patients who underwent an intrathecal continuous infusion catheter trial as an outpatient. The patients underwent an intrathecal catheter placement at the L2/3 interlaminar space with the catheter tip at $\mathrm{T} 10$, regardless of pathology. The patients were connected to an ambulatory pump and were monitored as an outpatient for up to 3 days.
Results: Ninety of the 98 patients had greater than $50 \%$ pain relief and proceeded to a permanent intrathecal pump implant. The average effective dose of morphine or hyrdromorphone was $480 \mathrm{mcg} /$ day. Using our outpatient and dosage methodology, 0 patients had serious adverse events which include infection, self-reported or caregiver-reported respiratory difficulty, or an emergency room (ER) visit during the trial.

The most common adverse events were postdural-puncture headache (PDPH), which occurred in $41 \%$ of the patients, nausea/vomiting in $28 \%$ of the patients, and catheter dislodgment in $18 \%$ of the patients.

Limitations: The sample size can be increased to further prove safety. A multicenter study would also prove to be a benefit.

Conclusion: Based on this study, we have devised a safe and efficacious methodology for performing an outpatient intrathecal trial using a continuous infusion catheter, using low doses. Implanted drug delivery systems are being increasingly utilized for chronic non-malignant pain, and this study implements a safe methodology. This study hopes to provide better patient access or an alternative to inpatient intrathecal infusion trials, which can be cumbersome.

Key words: Targeted drug delivery, pump trial, intrathecal opioid, intrathecal infusion, outpatient trial, chronic non cancer pain
From : Advanced Spine and Pain, Stafford, VA

Author for correspondence: Vipul and Mangal, MD

Address: Advanced Spine and Pain, 450 Garrisonville Rd, Ste 109, Stafford, VA 22554

E-mail: vmangal@gmail.com
Chronic pain is a significant public health challenge affecting nearly $30 \%$ of the population. It also presents a major economic burden and a sizable public health problem in industrialized countries $(1,2)$. Chronic pain can be attributed to various etiologies; however, cancer and non-cancer are the broad categories of 
classification. Non-cancer pain remains the underlying cause, accounting for the majority of cases presented for treatment with the use of targeted drug delivery systems (TDDS) $(3,4)$. The treatment, or more accurately the management, of chronic, severe, intractable pain of non-malignant etiology has eluded health care authorities for decades, ever since the introduction of TDDS by Medtronic in 1991; the use of such modality has been gaining popularity (5). Advances in understanding the mechanism of pain and response to analgesics, as well as recently introduced dosing strategies has led to better documented longterm outcomes in terms of pain relief and functional improvement (6-9)

Proper patient selection is crucial to achieving favorable outcomes when utilizing TDDS as a modality in the management of chronic intractable pain. Many authors, as well as payer parties, require the demonstration of a positive response to neuraxial administered analgesics prior to implantation of a TDDS (10). In a retrospective, multicenter analysis, various trial methods have been employed to screen patients that may potentially benefit from this form of therapy. These include single epidural or intrathecal injections and continuous infusions via epidural or intrathecal catheters (11). However, there is no published data to support the superiority of any single approach. A recent retrospective, multicenter survey of physicians in the US revealed that the most common method was a continuous intrathecal infusion (35\%), followed closely by bolus intrathecal injections (34\%), and bolus epidural injections (25\%) (11).

The Polyanlagesic Consensus Conference (PACC) 2012 guidelines recommend an inpatient trial for a continuous infusion intrathecal or epidural catheter or 23 hours of inpatient observation for a single-shot intrathecal injection of an opioid. The reasoning behind this is due to the risk of delayed respiratory depression, which requires close observation during the procedure. (12)

Hamza et al (13) published a prospective randomized, blinded study that demonstrates the lack of superiority comparing intrathecal boluses to intrathecal infusion trialing techniques prior to implantation of a TDDS, in terms of predicting trial success/failure and predicting long-term outcomes following the implant (13). In the study, trials were conducted in an inpatient setting. However, it was emphasized that in the absence of evidence supporting superiority of a specific technique, it is important to design a trial technique based on the resources available to every individual provider.

This study aims to provide a safe and effective methodology for an outpatient intrathecal infusion trial technique using low dose trialing techniques. With an outpatient methodology, this study aims to provide better patient access and an alternate methodology for a trialing technique compared to that of an inpatient trial, which can be cumbersome, expensive, time consuming for the physician, and not representative of the home environment, thus resulting in a hospital system, which may not be consistent with results in the patient's home environment and is not always necessary. With close monitoring available at home, by a family member or friend, 98 chronic non-cancer pain patients underwent an outpatient continuous infusion intrathecal catheter trial.

\section{METHODS}

The study was conducted retrospectively through medical chart review and patient survey analysis. In our community-based practice, 98 patients with severe chronic non-cancer pain, that failed to respond to multiple lines of conservative and interventional treatments, were referred to the practice for consideration of a TDDS implantation. Each patient underwent a detailed medical history and physical examination, with special attention to previous treatments. Imaging and electro-diagnostic studies were reviewed. These patients had failed conservative treatment options such as NSAIDs, opioids, injections, spinal cord stimulation, and surgeries. The patients underwent a surgical consultation to rule out the presence of any pain relevant to surgically correctable lesion. Any of the patients who were taking oral opioids had to wean to less than $100 \mathrm{mg}$ morphine equivalent prior to the trial.

Before the trial began, all of the patients received the following: educational material outlining TDDS therapy, a clinic handout explaining the protocol and rationale of TDDS therapy, and information explaining the method of trialing. In our study, 98 patients were found to be appropriate candidates for the therapy $(n=98)$. The inclusion criteria were defined as pa- 
tients who underwent outpatient intrathecal trial from January 1, 2014 to December 31, 2016. All of the patients had duration of symptoms greater than one year. Table 1 shows the demographics by gender.

Multiple patients had several of the diagnoses displayed in Table 2. Multiple patients had failed back surgery syndrome, chronic low back pain, and lumbar radiculopathy. The patients had to have failed physical therapy, medication management, injections such as epidural steroid injections, spinal cord stimulator therapy, and chronic intractable pain, as well as have symptoms lasting longer than one year to qualify to undergo an intrathecal pump trial. The patients were required to have a family/caretaker that could accompany and monitor them during the trial period. If there were any signs of adverse events, the patients were told to call the office or to immediately go to the emergency room (ER).

All of the patients were trialed at an outpatient clinic with an indwelling intrathecal catheter. The procedure entailed placing an intrathecal catheter using a Peri$\mathrm{fix}^{\circledR}$ Epidural Catheter/Touhy kit (B.Braun, Bethlehem, PA) under fluoroscopy at the L2/3 level on entry. The interlaminar space was accessed using a 17 gauge Touhy needle, and a 20 gauge catheter was then inserted, placing the catheter tip at T8-T10 using fluoroscopy for guidance, regardless of pathology or diagnosis. The catheter level placement was the same to provide consistency in the procedure for all of the patients. The catheter was secured to the patient and connected to a Curlin Medical External Infusion Pump (Curlin Medical, LLC, Huntington Beach, CA). The catheter was not tunneled, but secured to the skin using steristrips, gauze, and hypafix tape. The patients were started on an infusion of $100 \mathrm{mcg} / \mathrm{ml}$ morphine or hydromorphone at a rate of $0.1 \mathrm{~mL} /$ hour or $240 \mathrm{mcg} /$ day. The patients were instructed to discontinue any oral opioids during the trial and were discharged home into the care of a responsible care giver, such as a friend or a family member. The patients were then seen back in the clinic as an outpatient for 3 days, and the doses were adjusted accordingly, if needed, incrementally by $0.1 \mathrm{~mL} /$ hour or $240 \mathrm{mcg} /$ day to $480 \mathrm{mcg} /$ day, and then, if needed, to max dose of morphine or hydromorphone of 720 $\mathrm{mcg} /$ day based on patient outcomes. The median effective dose where the patients achieved success
Table 1. Patient demographics

\begin{tabular}{|l|c|}
\hline \multicolumn{2}{|c|}{ Demographics } \\
\hline Male & $38 \%$ \\
\hline Female & $62 \%$ \\
\hline Average Age & $52 \mathrm{yrs}$ \\
\hline
\end{tabular}

Table 2. Patient diagnoses

\begin{tabular}{|l|c|}
\hline \multicolumn{1}{|c|}{ Diagnosis } & Percentage \\
\hline Lumbar radiculopathy/low back pain & $44 \%$ \\
\hline Cervical radiculopathy & $13 \%$ \\
\hline Failed back surgery syndrome & $58 \%$ \\
\hline Neuropathic pain & $40 \%$ \\
\hline
\end{tabular}

in the trial was $480 \mathrm{mcg} /$ day hydromorphone and/or morphine. In our trialing protocol, 17 patients had trials done with morphine $(n=17)$, and 83 patients had trials done with hydromorphone $(n=83)$. The patients were instructed to not drive during the trial and were also given instructions to not manipulate the pump as it was placed in a carry-on bag. The pump has a lockout where the patient cannot adjust the dose themselves, and the pump operates independently. If there were any alarms or issues, the patient was instructed to call the office immediately. There was no home-care or home-infusion nursing involved during the trial. A successful trial was considered if a patient self-reported pain reduction by greater than $50 \%$ without taking any oral opioids.

\section{RESULTS}

A total of 98 patients were trialed $(n=98)$, and 90 patients had greater than $50 \%$ pain relief without taking any oral opioids and proceeded to the permanent implant $(n=90)$ (Table 3). A failed trial consisted of a patient who subjectively did not receive over $50 \%$ pain relief in their symptoms. The criteria for failed trial ( $8 \%$ of the patients) consisted of inadequate analgesia subjectively or intolerance of the medication.

Table 4 lists the adverse effects encountered in this study. The patients reported no serious adverse events, and 46 patients had any one or more of the common adverse events listed in Table 4, with post-dural-puncture headache (PDPH) being the 
Table 3. Percentage of patients that proceeded to the permanent implant

\begin{tabular}{|l|c|}
\hline $\begin{array}{l}\text { Patients that Proceeded to the } \\
\text { Permanent Implant }\end{array}$ & Percentage \\
\hline Permanent implant & $92 \%$ \\
\hline
\end{tabular}

Table 4. Adverse events

\begin{tabular}{|l|c|}
\hline \multicolumn{1}{|c|}{ Serious Adverse Events $(\mathbf{n}=\mathbf{0})$} & Percentage \\
\hline Infection & $0 \%$ \\
\hline ER visit & $0 \%$ \\
\hline $\begin{array}{l}\text { Subjective self-reported/caregiver-observed } \\
\text { respiratory difficulty }\end{array}$ & $0 \%$ \\
\hline Common Adverse Events $(\mathrm{n}=46)$ & \\
\hline PDPH & $41 \%$ \\
\hline Sedation/dysphoria & $5 \%$ \\
\hline Catheter dislodge & $18 \%$ \\
\hline Early discontinuation & $15 \%$ \\
\hline Nausea/vomiting & $28 \%$ \\
\hline
\end{tabular}

most common. The average age of patients having a PDPH was 49 years old. To resolve the PDPH, 10 of the patients required an epidural blood patch procedure $(n=10)$. Excessive sedation/dysphoria resolved within 12 hours of discontinuation of the trial. If a catheter dislodged from the intrathecal space, the entire trial was discontinued, due to the catheter no longer being in the intrathecal space. If the catheter dislodged from the original intrathecal space at T8$\mathrm{T} 10$, but the catheter was still in the intrathecal space, the catheter was reinforced at the skin and the trial was continued.

There were a multitude of reasons why a trial was discontinued early for 15 patients $(n=15)$. The most frequent cause was nausea/vomiting with PDPH, which occurred in 11 patients $(n=11)$. A total of 3 patients were discontinued early due to the catheter dislodging from the intrathecal space $(n=3)$. There was one patient that was discontinued early due to excessive sedation or dysphoria without respiratory depression $(n=1)$. Of the patients in which the trial was discontinued early, 13 out of the 15 patients proceeded with the permanent implantation. The patients were implanted because they had enough relief of their chronic symptoms to proceed with per- manent implantation, and the majority of the patients discontinued early due to the PDPH.

The dysphoria and sedation occurred when patients requested an increase from $480 \mathrm{mcg} / \mathrm{day}$ to $720 \mathrm{mcg} /$ day. Since the external Curlin Medical pump that was used cannot be adjusted to have a smaller dose titration, this increase led to sedation/dysphoria in $5 \%$ of the patients. The permanent intrathecal pump implant allows for finer incremental changes.

There were no patients with signs of fever, meningitis, cellulitis, or infection at the insertion site. The patients were not given any antibiotics prophylactically or during the course of trial. There were no ER visits during the trial, and the patients or their caregivers did not complain of or observe respiratory difficulty.

\section{DISCUSSION}

We are presenting a series of 98 patients who underwent a continuous intrathecal infusion trial of opioid via an indwelling intrathecal catheter prior to implantation of a TDDS for chronic, severe, intractable non-malignant pain. The trial was done on an outpatient basis and the data shows there were no serious adverse events such as infection, subjective respiratory difficulty, and/or ER visit. The patients were able to tolerate the trialing technique, and the common adverse events were similar to what is expected from an intrathecal infusion catheter via an inpatient technique. The common adverse events resolved at the end of the trial after discontinuation of the catheter, with a small percentage requiring an epidural blood patch, as expected, from a PDPH. In the future, the rate of occurrence of a PDPH can most likely be reduced by changing to an 18 gauge Touhy, with a 20 gauge catheter. With less of a diameter discrepancy, theoretically, there will be less cerebrospinal fluid leaks, leading to less incidence of a PDPH. In our opinion, the nausea/vomiting was also most likely due to the PDPH, as those symptoms typically occurred concurrently, and the patients who received the permanent implant did not have those symptoms with the same intrathecal drug.

Based on this study's results, we have devised an outpatient based trialing technique with an indwelling intrathecal catheter. In our series of 98 patients, we found this technique to be safe without significant ad- 
verse events and to be effective when done with low doses of hydromorphone or morphine, with no oral opioids during the trial. This study shows that trialing in an outpatient basis using a continuous intrathecal catheter with dosing less than $1 \mathrm{mg} /$ day is safe with morphine or hydromorphone. The common effective dose in the study was hydromorphone or morphine at $480 \mathrm{mcg} /$ day. In our opinion, the potency difference of hydromorphone and morphine isn't necessarily pertinent equate in the intrathecal space or at low doses, therefore both drugs were tested with similar dose regimens during the trial protocol. Respiratory depression and possible overdose were monitored by the patient and family member/caregiver during the trial. The risk of respiratory depression in any form of intrathecal trialing is present, but we believe by using a low dose trialing method, the risk is minimal, even with outpatient trialing. This method of trialing also provides a more accessible/suitable method for some physicians.

Implanted Intrathecal Drug Delivery Systems are being increasingly used for the treatment of chronic non-cancer pain, and have been well-established in the management of patients with intractable pain that is resistant to treatment with less invasive forms of therapy. The trialing procedure must be considered in the overall context of patient selection and management and, therefore, should be viewed as part of the overall process and not an isolated aspect of the therapy. It is important to construct a trial technique based on the patient's need, with emphasis on functional improvement; equally important is the available support system in terms of ancillary services, methods of observation, the need for intensive or stepdown units, the finances/cost-effectiveness of each method, and the level of expertise (8).

In a follow-up study, Hamza et al (13) presented a randomly assigned perspective comparison between 2 popular trialing techniques. Namely, intermittent intrathecal boluses and chronic intrathecal infusion as a screening test prior to implantation of a TDDS. Hamza concluded that there was no significant difference between the 2 driving techniques in terms of predicting trial success/failure and in terms of predicting the long-term outcome. Others concluded that both of the trialing techniques are closely similar as screening methods prior to implantation. The author also emphasized that the trialing modality should be dependent on the specific and local resources of the treating physician (13).

Proper patient selection is one of the crucial elements to be considered in preparation for TDDS implantation for chronic malignant pain. A major element in making the proper patient selection is a trial of intrathecal opioids prior to implantation of the system. In the US, as well as some European countries, it is required to perform such a trial via a temporary catheter. A trial is thought to provide an assessment of short-term pain relief, a guidance on therapeutic dosing regimen, and to determine if an individual can tolerate the proposed medication (14-18).

Some authors have challenged the grounds of performing a trial because the procedure has not been shown to predict long-term success of intrathecal therapy, and it is also virtually impossible to trial all possibly administered medications $(14,19)$.

However, Prager et al (20) as well as Doleys et al (21) have emphasized the need for a well-structured trial that is designed to provide an individualized opportunity to experience the potential analgesia, possible side effects, and more importantly, functional improvement. A positive response was adequate pain reduction, and improvement in function is considered a requirement prior to implantation of a TDDS.

Multiple techniques and methods have been utilized to perform screening tests for TDDS trials prior to implantation; those techniques include introducing opioids in either the epidural or intrathecal space. Intrathecal opioids may also be introduced through an interrupted bolus or chronic infusion (11), and it has been generally accepted that a reduction in the VATS by $50 \%$ without significant side effects is an acceptable cutoff limit for considering a trial to be positive, however, there is no one consensus regarding the length of the trial, discontinuation of the trial, or the taper of systemic opioids during trial (22-24).

Recently, a single-blinded, placebo-controlled, dose-escalating trialing method via an indwelling IT catheter was introduced by Hamza et al (8). In this study, the author prospectively studied 58 patients with a 3-year follow-up after the implant. Prior to the trial, patients' oral/systemic opioids were weaned by $50 \%$. An inpatient trial was done, as mentioned, 
using single opioid boluses, followed by placebo. A successful trial was defined as reduction in pain, improvement in function, lack of significant adverse events, and no report of improvement with the saline injection. A failed trial was defined as improvement with the saline injection that was superior to the opioid injection. In the study, 3 patients failed the trial and were not implanted. The remaining patients were implanted and reported significant improvement through the observation period. The authors introduced the technique as simple and easily performed, with no dose conversion, as well as a method to manage oral systemic opioids relative to pump implant.

To achieve optimal success, the treating physician should bear in mind the financial and logistical impacts, needs, and demands of performing a trial prior to an implantation of a TDDS. Therefore, we are presenting an outpatient trialing method with an indwelling intrathecal catheter as an option that might present less burden in terms of financial demands to the patient and physician, and an effective process to demonstrate pain relief for the patient adequately.
Our recommendations are titrating down patient oral opioid usage to less than $100 \mathrm{mg}$ morphine equivalent prior to trialing, and then performing the trial at a low dose with the patient discontinuing any oral opioids during the trial. This will not only optimize the trial, but will also optimize the therapy with the permanent implant. We also believe it offers the patient, as deemed appropriate, an opportunity to become an active participant in the care plan and, to an extent, the execution of this plan.

\section{Limitations}

A possible limitation is the use of a single center study, rather than a multicenter study. However, with the single study site it is relatively guaranteed that the specific protocol for overall management, instructions, messaging, and explaining these instructions to the patient has been emphasized, which can be a factor in obtaining better outcomes. Another limitation of this study might be the small number of patients, as the sample size will need to be significantly higher to ultimately prove the safety of this technique. 


\section{REFERENCES}

1. Manchikanti L, Boswell MV, Singh V, Benyamin RM, Fellows B, Abdi S, Buenaventura RM, Conn A, Datta S, Derby R, Falco FJ, Erhart S, Diwan S, Hayek SM, Helm S II, Parr AT, Schultz DM, Smith HS, Wolfer LR, Hirsch JA. Comprehensive evidencebased guidelines for interventional techniques in the management of chronic spinal pain. Pain Physician 2009; 12:699-802.

2. Gupta A, Mehdi A, Duwell M, Sinha A. Evidence-based review of the pharmacoeconomics related to the management of chronic nonmalignant pain. J Pain Palliat Care Pharmacother 2010; 24:152-156.

3. Manchikanti L, Singh V, Datta S, Cohen SP, Hirsch JA. Comprehensive review of epidemiology,scope, and impact of spinal pain. Pain Physician 2009; 12:E35-E70.

4. Dagenais S, Caro J, Haldeman S. A systematic review of low back pain cost of illness studies in the United States and internationally. Spine J 2008; 8:8-20.

5. Belverud S, Mogilner A, Schulder M.Intrathecal pumps. Neurotherapeutics 2008; 5:114-122.

6. YakshTL, RudyTA. Analgesia mediated by a direct spinal action of narcotics. Science 1976; 192:1357-1358.

7. Atweh SF, Kuhar MJ. Autoradiographic localization of opiate receptors in rat brain. I.Spinal cord and lower medulla. Brain Res 1977; 124:53-67.

8. Hamza M, Doleys D, Wells M, Weisbein J, Hoff J, Martin M, Soteropoulos C, Barreto J, Deschner S, Ketchum J. Prospective study of 3-year follow-up of low-dose intrathecal opioids in the management of chronic nonmalignant pain. Pain Med 2012; 13:1304-1313.

9. Grider JS, Etscheidt MA, Harned ME, Lee J, Smith B, Lamar C Bux A. Trialing and maintenance dosing using a low-dose intrathecal opioid method for chronic nonmalignant pain: A prospective 36-month study. Neuromodulation 2016; 19:206-219.

10. Krames ES. Intraspinal analgesia for nonmalignant pain. In: Waldman SD (ed). Interventional Pain Management. WB Saunders, Philadelphia 2001, pp 609-620.

11. Penn RD, Paice JA. Chronic intrathecal morphine for intractable pain. J Neurosurg 1987; 67:182-186.

12. Deer TR, Prager J, Levy R, Rathmell J, Buchser E, Burton A, Caraway D, Cousins M, De Andrés J, Diwan S, Erdek M, Grigsby E, Huntoon M, Jacobs MS, Kim P, Kumar K, Leong M, Liem L, McDowell GC II, Panchal S, Rauck R, Saulino M, Sitzman BT, Staats P, Stanton-Hicks M, Stearns L, Wallace M, Willis KD, Witt W, Yaksh T, Mekhail N. Polyanalgesic consensus conference 2012: Recommendations for management of pain by intrathecal drug delivery: A report from an interdisciplinary panel. Neuromodulation 2012; 15:436-64; discussion 464-6.

13. Hamza M, Doleys DM, Saleh IA, Medvedovsky A, Verdolin $\mathrm{MH}$, Hamza M. A prospective, randomized, single-blinded, head-to-head long-term outcome study, comparing intrathecal
(IT) boluses with continuous infusion trialing techniques prior to implantation of drug delivery systems (DDS) for the treatment of severe intractable chronic nonmalignant pain. Neuromodulation. 2015; 18:636-48; discussion 649.

14. Deer TR, Smith HS, Cousins M, Doleys DM, Levy RM, Rathmell JP, Staats PS, Wallace M, Webster LR. Consensus guidelines for the selection and implantation of patients with noncancer pain for intra-thecal drug delivery. Pain Physician 2010; 13:E175-E708.

15. Deer T, Krames ES, Hassenbusch SJ, Burton A, Caraway D, Dupen S, Eisenach J, Erdek M, Grigsby E, Kim P, Levy R, McDowell G, Mekhail N, Panchal S, Prager J, Rauck R, Saulino M, Sitzman T, Staats P, Stanton-Hicks M, Stearns L, Willis D, Witt W, Follett K, Huntoon M, Liem L, Rathmell J, Wallace M, Buchser E, Cousins M, Ver Donck A. Polyanalgesia consensus Conference 2007: Recommendations for the management of pain by intrathecal drug delivery: Report of an interdisciplinary expert panel. Neuromodulation 2007; 10:300-328.

16. National Coverage Determination (NCD) for Infusion Pumps. (280.14) CMS www.cms.gov/medicare-coverage-database/details/ncd-details.aspx NCDId=223\&ncdver=2\&SearchType=Ad vanced\&CoverageSelection $=$ National $\&$ NCSelection $=$ NCD\&K eyWord=Infusion+Pain+Pumps+\&KeyWordLookUp=Doc \&Ke yWordSearch Type=And\&kq=true \&bc=IAAAACAAAAAA\& (accessed 02/2016).

17. UnitedHealthcare www.unitedhealthcareonline.com/ccmcontent/Providerl//UHC/ en-US/Main\%20Menu/Tools $\% 20 \& \% 20$ Resources/Policies\%20and\%20Protocols/ Medicare\%20Advantage $\% 20$ Reimbursement $\% 20$ Policies/I/InfusionPumps_ NCD280-14_09262012.pdf (accessed 02/2016).

18. Anthem www.anthem.com/medicalpolicies/policies/mp_pw_ a053366.htm (accessed 02/2016).

19. Webster RL. Are intrathecal therapy trials necessary? Pract Pain Management 2007; 7:1541-1548.

20. Prager J, Deer T, Levy R, Bruel B, Buchser E, Caraway D, Cousins M, Jacobs M, McGlothlen G, Rauck R, Staats P, Stearns L. Best practices for intrathecal drug delivery for pain. Neuromodulation 2014; 17:345-372.

21. Doleys DM, Kraus JK. Psychological and addiction issues in intraspinal therapy. Semin Pain Med 2004; 2:46-52.

22. Krames ES, Olson KA. Clinical realities and economic considerations: Patient selection in intrathecal therapy. J Pain Symptom Manage 1997; 14 (Suppl.):S3-S13.

23. Prager JP. Neuraxial medication delivery: The development and maturity of a concept for treating chronic pain of spinal origin. Spine 2002; 27:2593-2605.

24. Prager J, Jacobs M. Evaluation of patients for implantable pain modalities: Medical and behavioral assessment. Clin J Pain 2001; 17:206-214. 
